\title{
Long-term survival after multimodal treatment involving radiotherapy for huge hepatocellular carcinoma with oligometastasis: a case report
}

\author{
Byung Min Lee, Jinsil Seong \\ Department of Radiation Oncology, Yonsei Cancer Center, Yonsei University College of Medicine, Seoul, Korea
}

Received May 31, 2021

Revised Jul. 30, 2021

Accepted Aug. 6, 2021
The clinical efficacy of local ablative treatment for oligometastasis is widely accepted in most cancers. However, due to limited data, this has not been the case for hepatocellular carcinoma (HCC). Here, we report a case of pulmonary oligometastasis of a huge HCC that was treated by multimodality with liver-directed concurrent chemoradiotherapy (CCRT) plus subsequent resection of the primary lesion and local ablative radiotherapy (RT) for subsequent lung oligometastatic lesions. In this patient, liver-directed CCRT induced significant tumor shrinkage with compensatory hypertrophy of the non-tumor liver, followed by curative resection. Surgical resection of the first and second pulmonary metastatic lesions as well as local ablative RT of the third lesion achieved complete tumor regression, which led to long-term survival of 6 years. Therefore, the active use of local ablative RT requires full consideration in cases of oligometastatic HCC. (J Liver Cancer 2021;21:163-168)

Keywords: Hepatocellular carcinoma; Metastasis; Radiotherapy; Combined modality therapy; Case report

\section{INTRODUCTION}

Oligometastasis, the transition from localized disease to widespread metastasis, is a widely accepted concept for various solid tumors. Local ablative treatments, including surgical resection or radiotherapy (RT), have been gaining attention for their ability to improve survival. Surgical intervention has been a standard treatment option for several decades. ${ }^{1}$ Furthermore, the local treatment of non-small cell lung cancer with oligometastasis was promising in a randomized phase II study. ${ }^{2}$ However, due to limited data, this has not been the case for hepato-

\footnotetext{
Corresponding author: Jinsil Seong

Department of Radiation Oncology, Yonsei Cancer Center, Yonsei University College of Medicine, 50-1 Yonsei-ro, Seodaemun-gu, Seoul 03722, Korea

Tel. +82-2-2228-8111, Fax. +82-2-2227-7823

E-mail: JSSEONG@yuhs.ac
}

cellular carcinoma (HCC); systemic treatment is the standard regardless of metastasis status. ${ }^{3,4}$ Here, we report a case of huge HCC treated with liver-directed concurrent chemoradiotherapy (CCRT), which reduced the tumor size and induced hypertrophy of the non-tumor liver. In subsequent lung metastasis, complete remission was achieved after two surgeries and RT. This case report was described according to CARE guidelines available from https://www.care-statement.org/.

\section{CASE REPORT}

A 30-year-old woman visited our clinic complaining of nausea and vomiting for 2-3 months. She had no history of viral hepatitis or underlying liver disease. She had no family history of HCC or other malignancies. As nausea and vomiting symptoms gradually worsened, she visited the hospital. 
Computed tomography (CT) revealed a 16-cm liver mass. On the initial complete blood count panel, the following were observed: a white blood cell count of $7.65 \times 10^{3} / \mu \mathrm{L}$, a hemoglobin level of $14.4 \mathrm{~g} / \mathrm{dL}$, and a platelet count of $2.03 \times 10^{3} / \mu \mathrm{L}$. A liver function test revealed a serum bilirubin level of $0.6 \mathrm{mg} / \mathrm{dL}$, an albumin level of $4.8 \mathrm{~g} / \mathrm{dL}$, and a prothrombin time international normalized ratio of 0.96 . Additionally, the serum alpha-fetoprotein (AFP) level was higher than 1.20x $10^{6} \mathrm{ng} / \mathrm{mL}$, while the protein induced by vitamin $\mathrm{K}$ absence or antagonist-II (PIVKA-II) was 1,371 mAU/mL.

Abdominal dynamic CT and magnetic resonance imaging (MRI) revealed a 16-cm hepatic mass in the left lobe with

\section{Pre-CCRT}

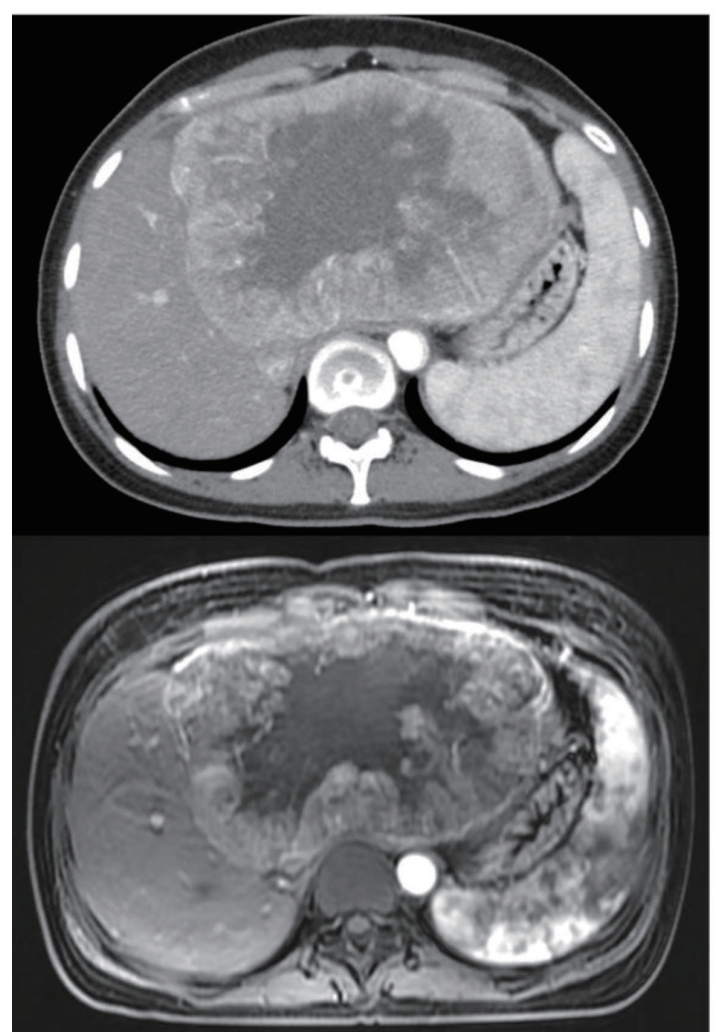

Post-CCRT

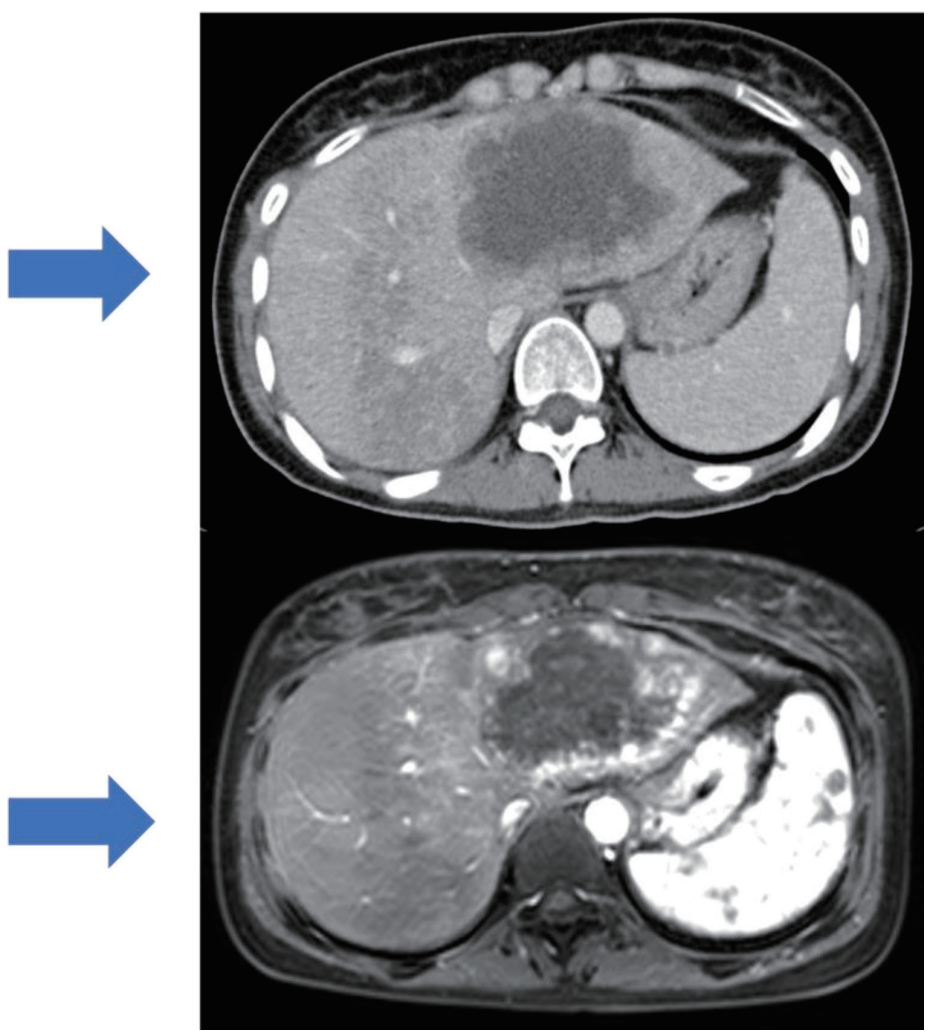

Figure 1. Liver dynamic computed tomography (top) and magnetic resonance imaging (bottom) findings before versus after liver-directed concurrent chemoradiotherapy (CCRT).
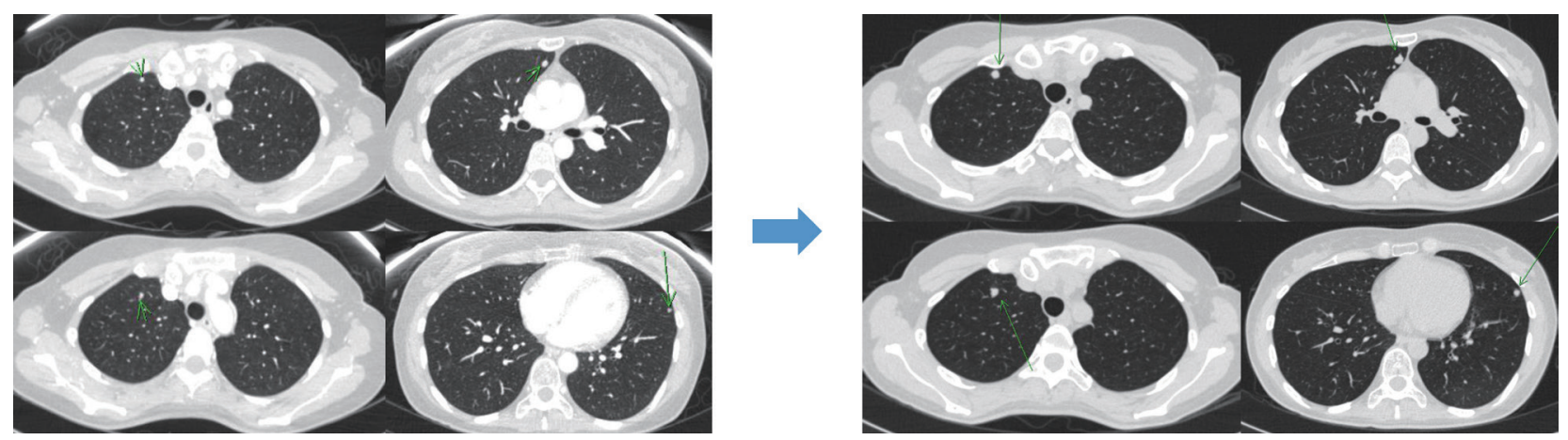

Figure 2. Chest computed tomography images showing the first pulmonary metastatic lesions before and after systemic treatment. 
typical radiological features, including enhancement in the arterial phase and washout in the portal and delayed phases. Neither intrahepatic metastasis nor lymph node enlargement was observed in the imaging study. Positron emission tomography-CT and chest CT showed no extrahepatic tumor metastasis.

Based on the clinical and imaging findings, the patient was diagnosed with a 16-cm HCC without extrahepatic metastasis. She had a Child-Pugh score of 5 and a performance status score of 0 . HCC was classified as an intermediate stage by the Barcelona Clinic Liver Cancer system and as Stage II (T2N0M0) by the modified Union for International Cancer Control TNM classification. Neoadjuvant CCRT was administered. In our institution, we performed CCRT for HCC involving local RT in conventional fractionation and concurrent hepatic artery infusion chemotherapy. The RT involved $46.8 \mathrm{~Gy}$ in 26 fractions, including the left liver mass. Hepatic arterial 5 -fluorouracil was administered during the first and fourth weeks of RT at a dose of $500 \mathrm{mg} /$ day.

After 1 month of CCRT, CT and MRI showed a decrease in HCC size (Fig. 1). Serum tumor marker levels also markedly decreased after CCRT. Serum AFP and PIVKA-II levels dramatically decreased from $>1.20 \times 10^{6}$ to $12,863 \mathrm{ng} / \mathrm{mL}$ and from 1,371 to $53 \mathrm{mAU} / \mathrm{mL}$, respectively. CCRT successfully reduced tumor size and resulted in hypertrophy of the nontumor liver. The patient underwent surgery after CCRT, comprising an extended left hepatectomy and caudate lobectomy. The tumor was approximately $8 \mathrm{~cm}$ in size with $70 \%$ tumor necrosis. She developed pulmonary metastasis 7 months after surgery. Chest CT revealed three metastatic lesions in the right lung and one metastatic nodule in the left lung (Fig. 2). After the lung metastatic lesions were identified, systemic treatment with sorafenib was initiated. After 9 months of administration, follow-up chest CT revealed that the metastatic lesions had increased in size. In addition, her serum tumor marker levels gradually increased. Serum

\section{Before salvage SBRT}
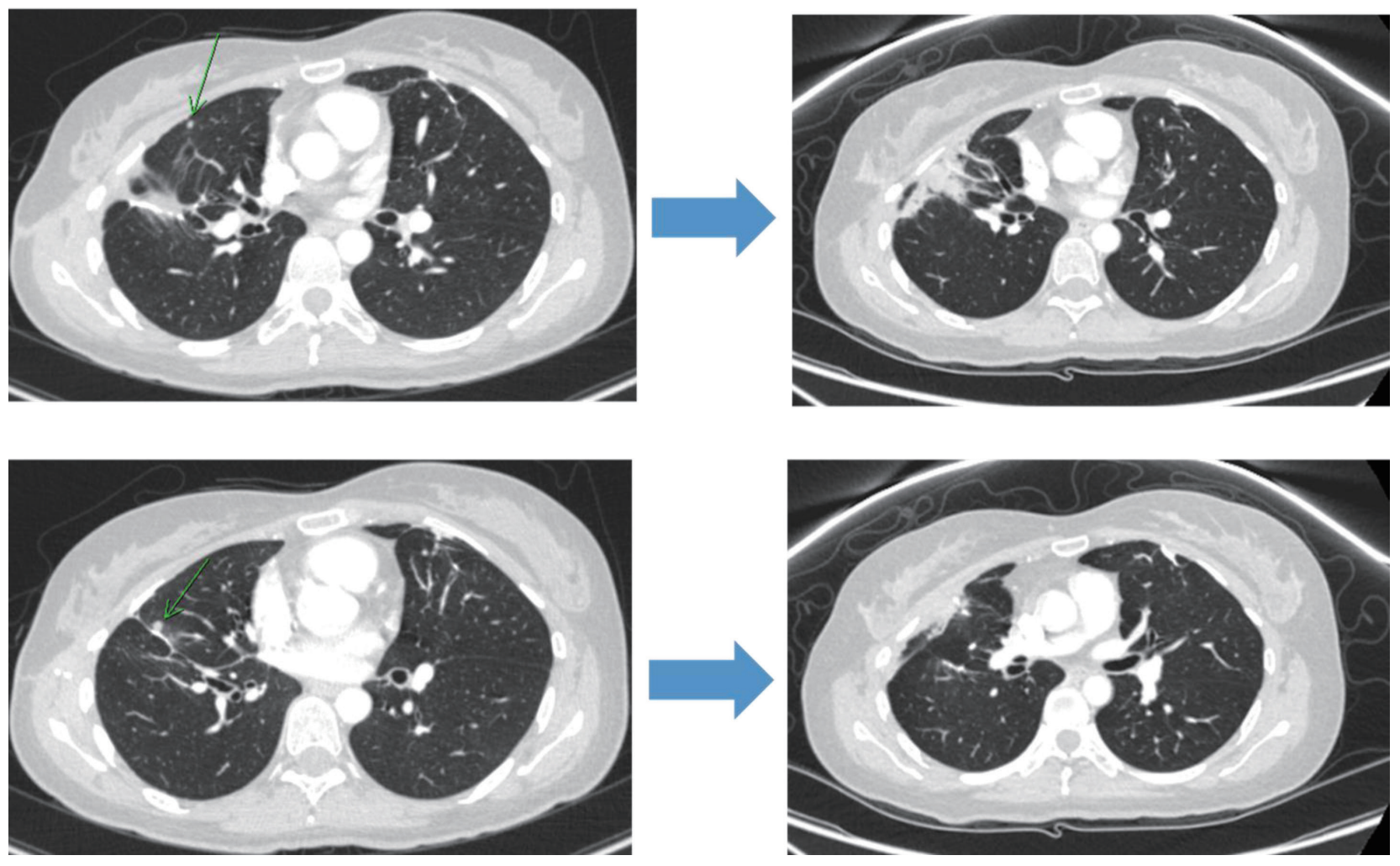

Figure 3. Chest computed tomography images showing pulmonary metastatic lesions before versus after stereotactic body radiotherapy (SBRT).

\section{4-year after SBRT}

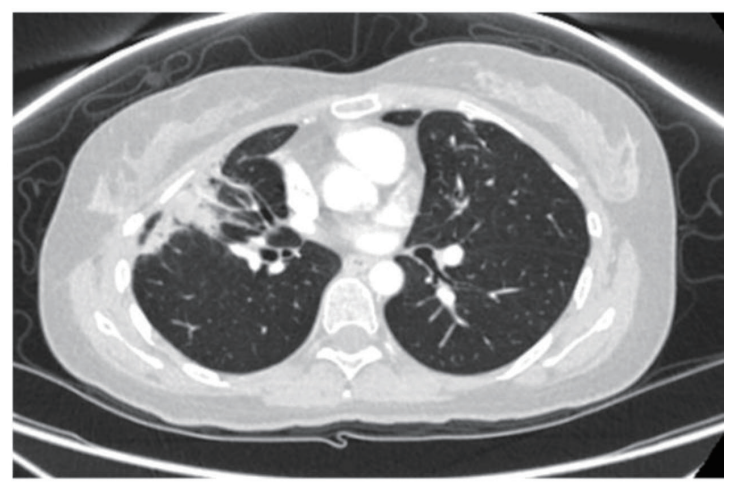


AFP was elevated from $367 \mathrm{ng} / \mathrm{mL}$ to $737 \mathrm{ng} / \mathrm{mL}$, while PIVKA-II increased from 103 to $174 \mathrm{mAU} / \mathrm{mL}$.

Left upper lobe wedge resection and metastasectomy of the right lung were performed. Immediately after the surgery, another metastatic lesion in the right lower lobe was observed on follow-up chest CT. Systemic chemotherapy with 5-fluorouracil and cisplatin was subsequently administered. After six cycles of administration, the metastatic lesion slightly decreased in size; a right lower lobe wedge resection was performed. Immediately after the surgery, two small metastatic nodules adjacent to the previous wedge resection site were observed on chest CT. Due to the previous surgeries, the patient and physicians preferred a nonsurgical treatment with stereotactic body RT (SBRT) to treat the lung nodules, which was administered by delivering $60 \mathrm{~Gy}$ in four fractions every other day. After SBRT, there was no evidence of a newly developed metastatic lesion with post-RT changes in the right upper lobe (Fig. 3). Furthermore, serum tumor markers gradually decreased and normalized 1 month after SBRT. Changes in tumor markers during follow-up are shown in Fig. 4. To date, there has been no disease recurrence during the 6-year follow-up period.

\section{Ethics statement}

This study was approved by the Institutional Review Board (IRB number: 4-2021-0788). The requirement for informed consent from patients was waived.

\section{DISCUSSION}

Here, we present a case of pulmonary oligometastasis from a large HCC. The patient was treated with multimodality with liver-directed CCRT with resection, local ablative RT, and metastasectomy for the subsequently developed lung oligometastatic lesions. Liver-directed CCRT led to tumor shrinkage and hypertrophy of the non-tumor liver for compensation, which enabled curative resection. Surgical resection was performed twice, while local ablative RT was performed once for pulmonary oligometastatic lesions after two surgeries. After confirming the progression of metastatic nodules in the lung, the patient opted to receive a local ablative treatment during a multidisciplinary team discussion for the following reasons. The patient was already on sorafenib as a systemic treatment, which means that treatment options were already limited. In addition, the patient was young and had good performance status. Her lesions were resectable, and she had adequate lung function. Surgical resection was

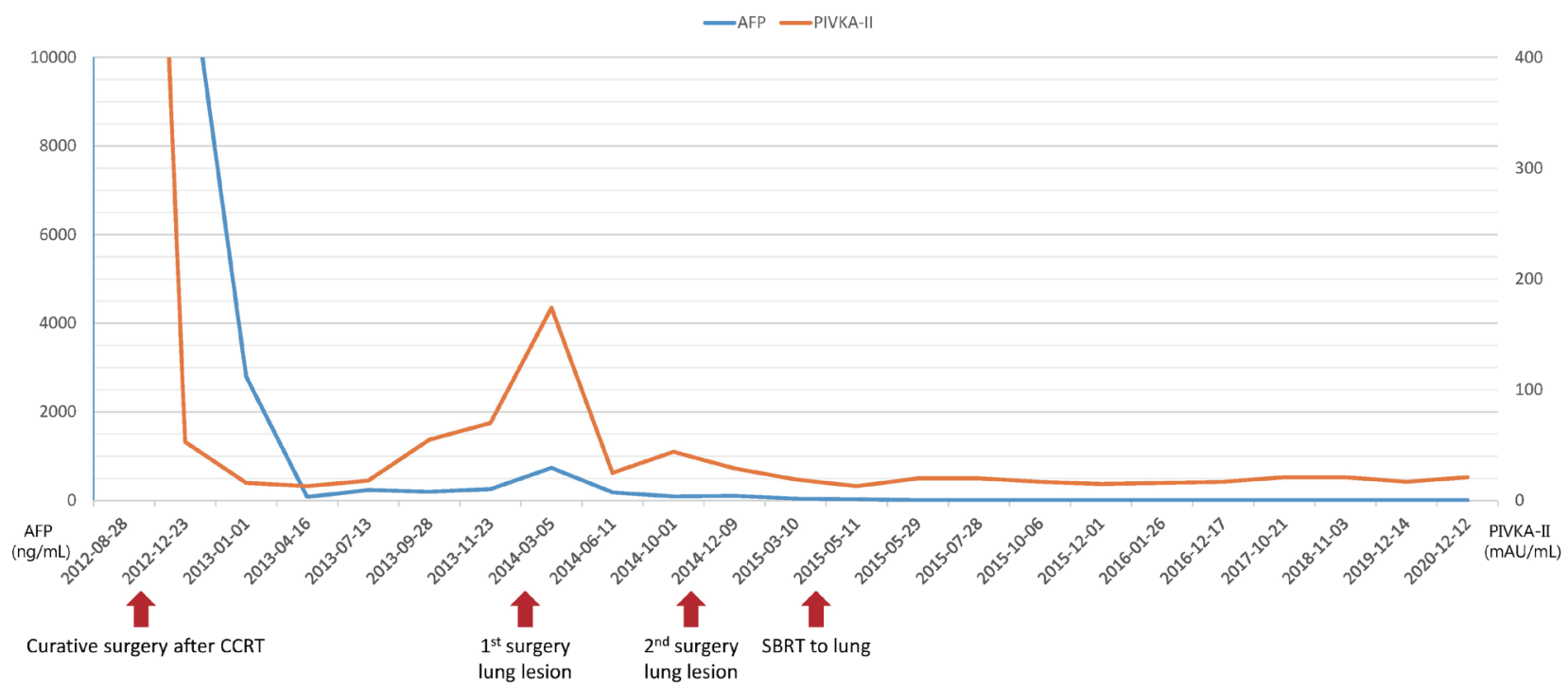

Figure 4. Alpha-fetoprotein (AFP) and prothrombin induced by vitamin K absence-II (PIVKA-II) levels during follow-up. CCRT, concurrent chemoradiotherapy; SBRT, stereotactic body radiotherapy. 
performed for the first and second newly occurring pulmonary metastases. However, for the third pulmonary metastasis, SBRT was administered because of several previously performed surgeries. Local treatment of pulmonary oligometastasis led to long-term (6-year) survival.

Treatments for limited metastatic lesions from HCC have lacking consensus and data. Local ablative treatments, including surgical resection and RT, have demonstrated curative effects for oligometastasis in various malignancies. ${ }^{1,2}$ The resection of hepatic oligometastatic lesions from colorectal cancer shows promising survival. ${ }^{1}$ In addition, local treatment for oligometastasis of non-small cell lung cancer has shown prolonged time-to-progression. ${ }^{2}$ Moreover, there are limited data regarding the local treatment of oligometastasis in HCC. One retrospective study demonstrated that local treatment for pulmonary oligometastasis from HCC improved patient survival. ${ }^{5}$ Active local treatment, including surgery and RT, enhanced oncologic outcomes in pulmonary oligometastasis, especially in patients with good liver function. Further studies with a larger number of patients are needed to confirm the significance of local treatment for HCC.

Managing primary liver lesions is a vital issue in locally advanced HCC. In this case, the patient had a $16-\mathrm{cm}$ HCC upon diagnosis, although successful primary cancer control was achieved through liver-directed multi-modality treatment. Our data demonstrated that liver-directed CCRT led to curative surgery and excellent survival outcomes. ${ }^{6}$ Curative surgery (surgical resection or liver transplantation) is the standard curative treatment for early HCC. However, the patient in this report had unresectable locally advanced HCC with vascular invasion upon diagnosis. Our data previously confirmed that liver-directed CCRT could be a bridge to curative surgery by converting the tumors within the Milan criteria. Patients who underwent surgery after CCRT showed excellent overall and progression-free survival rates despite the known dismal prognosis of advanced HCC.

5 -flurouracil is a key anti-cancer chemotherapy used to treat solid tumors, including HCC. It has been used alone or in combination with other chemotherapeutic drugs for HCC due to its relatively high response rate. ${ }^{7,8}$ Our patient showed a satisfactory treatment response to 5-flurouracil. Several studies have confirmed that the response rate to 5 -flurouracil is high and that survival is prolonged. Thus, response to 5-flurouracil could be used as a surrogate marker for patient survival. ${ }^{9}$

Even for pulmonary metastasis, patients were treated locally and systemically. Combined local and systemic treatment achieved complete regression of pulmonary metastasis and enabled long-term patient survival. Therefore, the active use of multimodality treatment requires consideration, especially in cases of locally advanced HCC with oligometastasis.

In this case, liver-directed CCRT decreased tumor size and enabled curative resection. Even after subsequent pulmonary metastasis, local treatment was performed and long-term survival was consequently achieved. This case demonstrates the importance of the active use of multimodality treatment for locally advanced HCC. However, to date, there is no consensus on the management of HCC with oligometastasis. Therefore, this treatment cannot be generalized to other patients. Further studies are still needed to confirm its importance and to identify patients who may benefit from these local treatments.

\section{Conflicts of Interest}

The authors have no conflicts of interest to disclose.

\section{Ethics Statement}

This study was approved by the Institutional Review Board (IRB number: 4-2021-0788). The requirement for informed consent from patients was waived.

\section{Funding Statement}

No funding to declare.

\section{Data Availability}

Data sharing not applicable to this article as no datasets were generated or analyzed during the study.

\section{ORCID}

Byung Min Lee https://orcid.org/0000-0002-5970-9773

Jinsil Seong https://orcid.org/0000-0003-1794-5951 


\section{Author Contribution}

Conceptualization: BML, JS.

Data curation: BML,JS

Methodology: JS

Project administration: JS

Writing original draft: BML

Writing review \& editing: IS

Approval of final manuscript: all authors.

\section{References}

1. Timmerman RD, Bizekis CS, Pass HI, Fong Y, Dupuy DE, Dawson LA, et al. Local surgical, ablative, and radiation treatment of metastases. CA Cancer J Clin 2009;59:145-170.

2. Gomez DR, Blumenschein GR Jr, Lee JJ, Hernandez M, Ye R, Camidge DR, et al. Local consolidative therapy versus maintenance therapy or observation for patients with oligometastatic nonsmall-cell lung cancer without progression after first-line systemic therapy: a multicentre, randomised, controlled, phase 2 study. Lancet Oncol 2016;17:1672-1682.

3. European Association for the Study of the Liver. EASL clinical practice guidelines: management of hepatocellular carcinoma. J
Hepatol 2018;69:182-236.

4. Heimbach JK, Kulik LM, Finn RS, Sirlin CB, Abecassis MM, Roberts $L R$, et al. AASLD guidelines for the treatment of hepatocellular carcinoma. Hepatology 2018;67:358-380.

5. Kim K, Kim TH, Kim TH, Seong J. Efficacy of local therapy for oligometastatic hepatocellular carcinoma: a propensity score matched analysis. J Hepatocell Carcinoma 2021;8:35-44.

6. Lee WH, Byun HK, Choi JS, Choi GH, Han DH, Joo DJ, et al. Liverdirected combined radiotherapy as a bridge to curative surgery in locally advanced hepatocellular carcinoma beyond the Milan criteria. Radiother Oncol 2020;152:1-7.

7. Ando E, Tanaka M, Yamashita F, Kuromatsu R, Yutani S, Fukumori $K$, et al. Hepatic arterial infusion chemotherapy for advanced hepatocellular carcinoma with portal vein tumor thrombosis: analysis of 48 cases. Cancer 2002;95:588-595.

8. Kim DY, Ahn SH, Kim SU, Choi SB, Lee KH, Park MS, et al. Adjuvant hepatic arterial infusional chemotherapy with 5 -fluorouracil and cisplatin after curative resection of hepatocellular carcinoma. Oncology 2011;81:184-191.

9. Nouso K, Miyahara K, Uchida D, Kuwaki K, Izumi N, Omata M, et al. Effect of hepatic arterial infusion chemotherapy of 5-fluorouracil and cisplatin for advanced hepatocellular carcinoma in the nationwide survey of primary liver cancer in Japan. $\mathrm{Br} J$ Cancer 2013:109:1904-1907. 